\title{
Model-Updated Image-Guided Neurosurgery: Preliminary Analysis Using Intraoperative MR
}

\author{
Michael I. Miga ${ }^{1}$, Andreas Staubert ${ }^{3}$, Keith D. Paulsen ${ }^{1,2}$, \\ Francis E. Kennedy ${ }^{1}$, Volker M. Tronnier ${ }^{3}$, David W. Roberts ${ }^{2}$, Alex Hartov ${ }^{1}$, \\ Leah A. Platenik ${ }^{1}$, and Karen E. Lunn ${ }^{1}$ \\ 1 Dartmouth College, Thayer School of Engineering, HB8000, Hanover, NH 03755 \\ \{michael.miga, keith.paulsen\}@dartmouth.edu \\ 2 Dartmouth Hitchcock Medical Center, Lebanon, NH 03756 \\ 3 University Hospital, Department of Neurological Surgery, Heidelberg School of \\ Medicine, Im Neuenheimer Feld 400, D-69120 Heidelberg, Germany.
}

\begin{abstract}
In this paper, initial clinical data from an intraoperative MR system are compared to calculations made by a three-dimensional finite element model of brain deformation. The preoperative and intraoperative MR data was collected on a patient undergoing a resection of an astrocytoma, grade 3 with non-enhancing and enhancing regions. The image volumes were co-registered and cortical displacements as well as subsurface structure movements were measured retrospectively. These data were then compared to model predictions undergoing intraoperative conditions of gravity and simulated tumor decompression. Computed results demonstrate that gravity and decompression effects account for approximately $40 \%$ and $30 \%$, respectively, totaling a $70 \%$ recovery of shifting structures with the model. The results also suggest that a non-uniform decompressive stress distribution may be present during tumor resection. Based on this preliminary experience, model predictions constrained by intraoperative surface data appear to be a promising avenue for correcting brain shift during surgery. However, additional clinical cases where volumetric intraoperative MR data is available are needed to improve the understanding of tissue mechanics during resection.
\end{abstract}

Keywords: finite element modeling and simulation, image guided therapy, intraoperative image registration techniques

\section{Introduction}

Over the past 10 years, there has been a signficant effort to understand the nature and extent of brain deformations during neurosurgery. The results of these investigations have suggested that intraoperative tissue movement may compromise the fidelity of preoperative-based image guidance if left unchecked [1], [2]. Recently, there has been a concerted effort by many investigators to augment existing neuronavigation systems to account for intraoperative brain deformation. Several medical centers have adopted the strategy of using intraoperative 
magnetic resonance (MR) imaging [3], [4]. Recent reports by Knauth et al. have systematically demonstrated in a thirty-eight patient study that intraoperatively updated image guidance significantly increased the amount of complete tumor removal from $37 \%$ to nearly $76 \%$ [5]. They have also illustrated potential problems with intraoperative MR imaging related to surgically-induced contrast enhancement which can be confused with contrast-enhancing residual tumor [6]. Others continue to question the use of intraoperative MR citing the fate of past experiences with intraoperative computed tomography in the 1980's. Moreover, intraoperative computed tomography did not face the OR-compatibility challenges of MR, yet it has not become a standard component of today's surgical "armamentarium" [7]. In addition, there is significant concern that the tumordefining boundary shown in MR is not representative of the complete tumor infiltration [7]. Appropriately, investigators are still determining the efficacy of intraoperative MR in order to identify its most important uses [8], [9].

Nonetheless, the need for intraoperative updating seems clear although the paradigm to provide such intraoperative data is somewhat more elusive. As an alternative to full volume imaging in the OR, we are developing a strategy that uses sparse intraoperative data (i.e. cortical shift tracking, coregistered intraoperative ultrasound) to drive a computational model that serves as the source for intraoperative image updates [10]. In vivo studies in controlled animal experiments have demonstrated the quantitative feasibility of this approach [11]. Preliminary experiments with humans experiencing intraoperative gravity-induced deformation suggest the model accuracy is comparable to that obtained in animal studies [12]. The advantages of such a strategy are its low cost and minimal impact into an already overcrowded OR environment. Additional impetus for exploiting computational models in the operating room (OR) is provided by the abundance of preoperative data that cannot be updated practically during surgery (i.e. positron emission tomography, electroencephalography, functional MR imaging, and MR spectroscopy). Computational models would be a natural choice to register this data with the updated image database.

A critical step in establishing the utility of computational models for image updating in the OR is to assess model predictions in light of full volume intraoperative image data when available. In this paper, preliminary results are presented from a clinical case having coregistered MR images pre- and immediatelypost tumor resection. Results demonstrate the potential predictive capability of models and provide valuable insight regarding the tumor-induced deformation arising from excision.

\section{Methods}

\subsection{Experimental Methods}

The patient's history reflected an astrocytoma grade 3 with non-enhancing and enhancing regions in the left pre-central region of the brain. For surgical planning and intraoperative neuronavigation, a preoperative MRI-data set was acquired (T1-FLASH 3D-seq., TR=34.0ms, TE=12.0ms, No.Acq.=1, TA=13:59, 

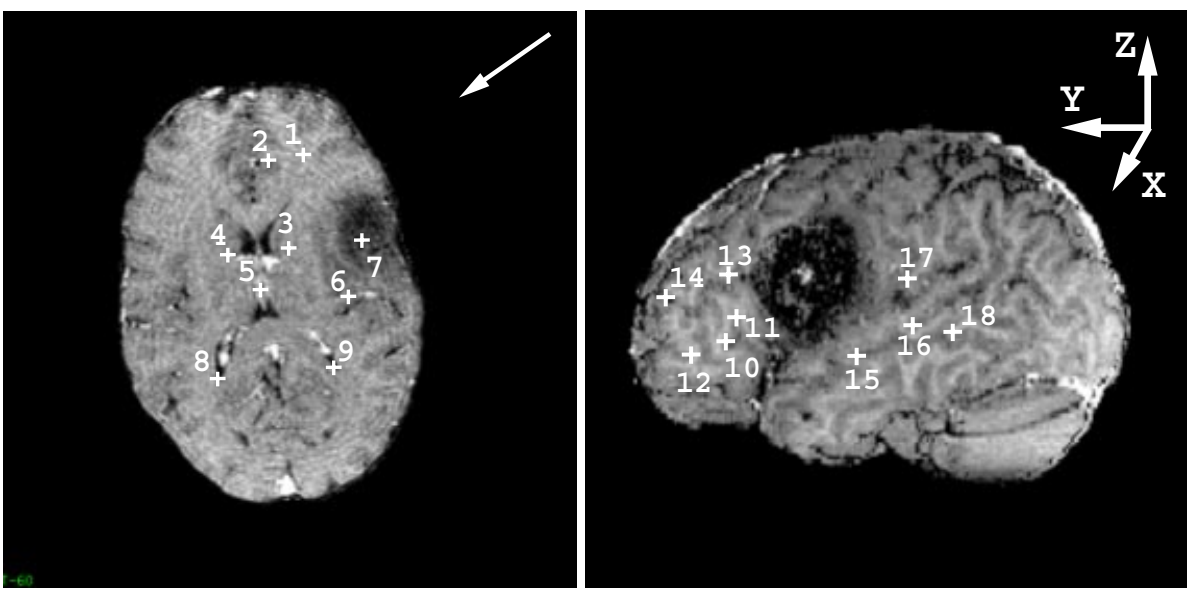

Fig. 1. Subsurface (left) and surface (right) point distribution for deformation tracking. Subsurface points $(1,2,3,4)$ are located more superior at higher elevations relative to the direction of gravity (designated with white arrow in left image), $(5,8,9)$ are located in-plane with tumor and $(6,7)$ are inferior to tumor.

$\mathrm{FOV}=260$, matrix $=256^{*} 256$, No.Sl. $=128$, Sl.Thk. $\left.1.4 \mathrm{~mm}\right)$ using an open lowfield MRI-scanner (Magnetom OPEN 0.2T, Siemens, Erlangen, Germany). The patient was brought to the OR and the patient's head was fixed in a dedicated head-holder coil for intraoperative MRI with head rotated to the right approximately 55 degrees. For neuronavigation the MKM-microscope (Zeiss, Oberkochen, Germany) was used, and the accuracy of patient registration with the preoperative MRI-data set was $1.7 \mathrm{~mm}$. The tumor was resected to the planned extent using the preoperative information. Of note, the margins of the pathologic structure were not clearly recognizable to the surgeon during resection. After total resection according to neuronavigation, the patient was transferred to the intraoperative MRI scanner adjacent to the OR using a dedicated patient transport system [13]. Intraoperative imaging in the Magnetom OPEN was performed using T1- and T2- weighted SE-sequences for diagnostic purposes and a T1-FLASH 3D-sequence (see above) for an intraoperative neuronavigational update [14],[4]. Intraoperatively, no residual enhancing regions of the tumor were found in the post-contrast media T1 images. Qualitative comparison of pre- and intraoperative images also indicated a complete tumor resection, but absolute quantitative estimations were not possible due to a regular brain-shift of approximately $1 \mathrm{~cm}$ around the resection cavity. The patient was transferred back to the OR, and the surgical access was closed using standard neurosurgical techniques.

Using this series of scans, we were able to identify 9 subsurface points and 9 cortical surface locations which could be tracked in both preoperative and intraoperative MR scans. Figure 1 illustrates the distribution of subsurface (left) and surface (right) points. We note that the subsurface distribution shown in 


\begin{tabular}{|l|l|ccc|c||c|l|ccc|c|}
\hline \hline$\#$ & Descr. & $\mathrm{dx}$ & $\mathrm{dy}$ & $\mathrm{dz}$ & $\mathrm{D}$ & $\#$ & Descr. & $\mathrm{dx}$ & $\mathrm{dy}$ & $\mathrm{dz}$ & $\mathrm{D}$ \\
\hline \hline 1 & Ant. Cortex & -4.0 & -6.0 & -1.3 & 7.3 & 10 & Ant. Sulcus & -5.0 & -7.0 & 0.0 & 8.6 \\
\hline 2 & Ant. Midln Cortex & -1.0 & -1.0 & -2.6 & 3.0 & 11 & Ant. Sulcus & -5.0 & -8.0 & 0.0 & 9.4 \\
\hline 3 & Ant. L. Lat. Horn & -1.0 & -2.0 & -1.3 & 2.6 & 12 & Ant. Sulcus & -3.0 & -6.0 & 1.3 & 6.8 \\
\hline 4 & Ant. R. Lat. Horn & -2.0 & -1.0 & -1.3 & 2.6 & 13 & Ant. Sulcus & -5.0 & -10.0 & -2.6 & 11.5 \\
\hline 5 & 3rd Vent. & -1.0 & 0.0 & 0.0 & 1.0 & 14 & Ant. Sulcus & -4.0 & -6.0 & 0.0 & 7.2 \\
\hline 6 & Post. Insular & 1.0 & 2.0 & 2.6 & 3.4 & 15 & Post. Sulcus & 2.0 & 3.0 & 2.6 & 4.4 \\
\hline 7 & Post. Insular & -2.0 & 1.0 & 2.6 & 3.4 & 16 & Post. Sulcus & 0.0 & 2.0 & 2.6 & 3.3 \\
\hline 8 & Post. R. Lat. Horn & -1.0 & 1.0 & 0.0 & 1.4 & 17 & Post. Sulcus & 1.0 & 1.0 & -1.3 & 1.9 \\
\hline 9 & Post. L. Lat. Horn & 0.0 & -1.0 & -1.3 & 1.6 & 18 & Post. Sulcus & 0.0 & 1.0 & 0.0 & 1.0 \\
\hline
\end{tabular}

Table 1. Measured brain feature displacement in millimeters in cartesian coordinates. $\mathrm{D}$ is the total displacement magnitude.

Figure 1 is not coplanar but in fact the points are distributed superior, in-plane, and inferior to the tumor location. Table 1 quantifies the deformations of these points as tracked between preoperative and intraoperative MR.

\subsection{Computational Methods}

We have chosen consolidation physics to represent deformation characteristics of the brain [15]. The governing equation describing mechanical equilibrium is,

$$
\nabla \cdot G \nabla \mathbf{u}+\frac{G}{1-2 \nu} \nabla \varepsilon-\alpha \nabla p+\left(\rho_{t}-\rho_{f}\right) \mathbf{g}=0 .
$$

The description is completed by a mass conservation equation relating volumetric strain to fluid drainage shown here,

$$
\nabla \cdot k \nabla p-\alpha \frac{\partial \varepsilon}{\partial t}-\frac{1}{S} \frac{\partial p}{\partial t}=0
$$

The mathematical framework of coupled equations (1) and (2) has been previously reported in detail [16], [17]. In previous work, material property studies have been reported [18], [19]. In the model's current form, the brain is treated as a homogeneous structure with respect to stiffness properties and heterogeneous with respect to its hydraulic behavior (i.e. $G_{w}=G_{g}=725 \mathrm{~Pa}, \nu_{w}=\nu_{g}=0.45$, $\alpha_{w}=\alpha_{g}=1.0, \frac{1}{S}_{w}=\frac{1}{S}{ }_{g}=0.0, k_{w}=1.0 \times 10^{-10} \frac{\mathrm{m}^{3} \mathrm{~s}}{\mathrm{~kg}}, k_{g}=5.0 \times 10^{-12} \frac{\mathrm{m}^{3} \mathrm{~s}}{\mathrm{~kg}}$, and $\rho_{w}=\rho_{g}=1020 \frac{\mathrm{kg}}{\mathrm{m}^{3}}$ where $w$ and $g$ refer to white matter and gray matter, respectively).

Figure 2a-c illustrate the assumptions regarding boundary conditions and surgical forces. Figure 2 a shows the computational geometry which consisted of 21,452 nodes and 113,044 elements. The exterior brain boundary conditions (Figure 2b) are of two types: stress-free (above dashed line) and slippage (below dashed line). Figure $2 \mathrm{~b}$ also shows the approximate intraoperative brain rotation with gravitational forces represented by a change in surrounding fluid 

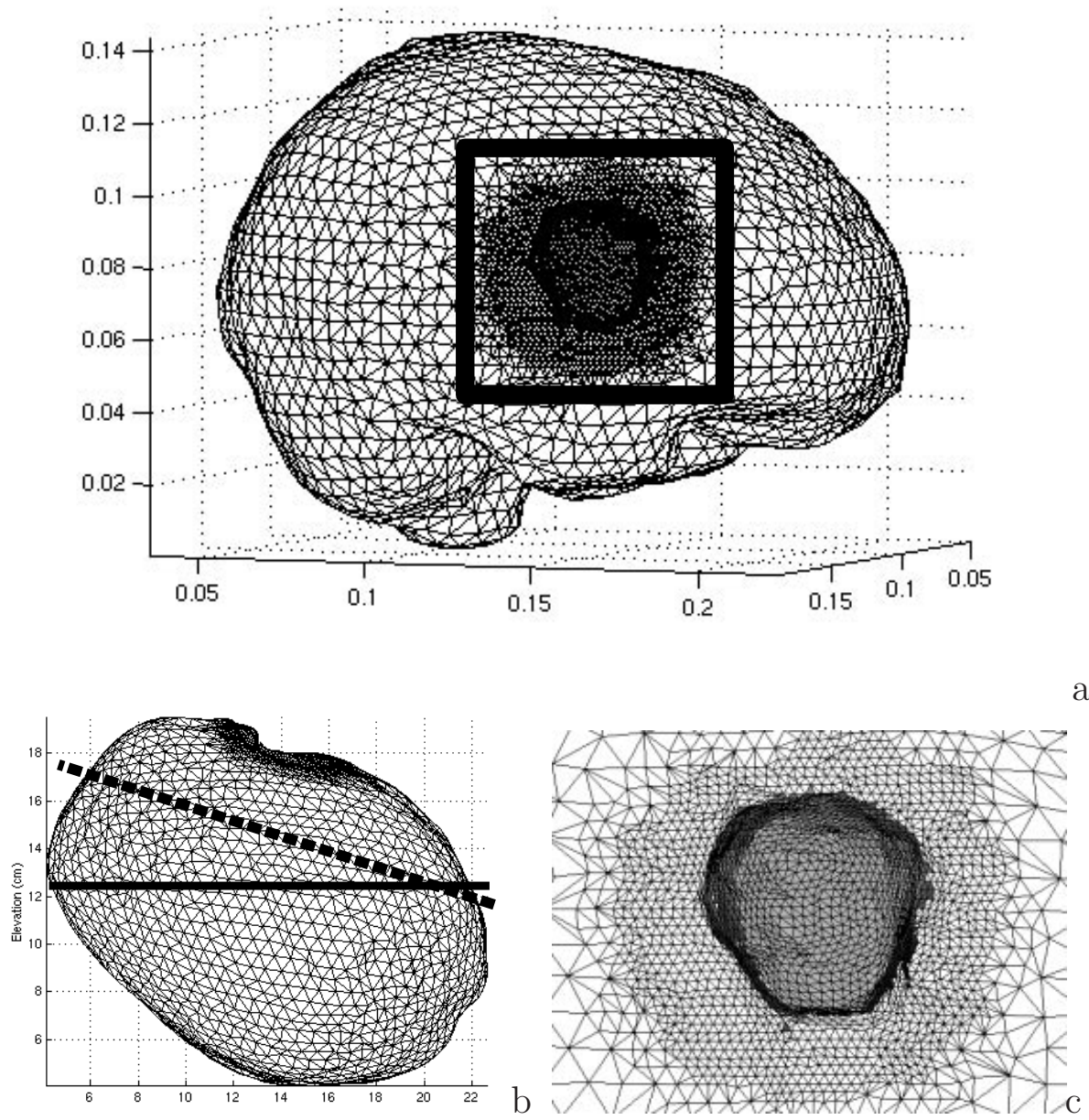

Fig. 2. Shown is the (a) computational geometry with boundary conditions for (b) brain exterior and (c) zoomed view of tumor cavity region designated in (a). In (b), head tilt with respect to gravity shown with solid and dashed line representing air/CSF and free/sliding surfaces respectively. In (c), the subcortical base of the cavity (central area in light grey) has a prescribed normal stress with the walls (dark grey vertical wall perimeter of region) given a larger radially in-plane driving stress distribution. 
density for elements above the air/cerebrospinal fluid (CSF) line (solid line). In Figure 2c, we have designated a new boundary condition to simulate the decompression of tissue following the removal of a tumor. Based on the preoperative/intraoperative data, we have approximated the removed tumor mass and deleted this tissue from our model. To simulate decompression, we have applied a non-uniform stress distribution to account for the relaxation of compressive forces previously induced by tumor growth. At the subcortical base of the cavity, the decompressive stress is directed normally and is approximately $5 \mathrm{~mm} \mathrm{Hg}$. Along the perimeter walls of the cavity, stress has been directed radially inward towards the center of the cavity in a plane approximately tangent to the brain suface. The stress distribution values ranged from 5 to $17.5 \mathrm{~mm} \mathrm{mg}$. The impetus for this non-uniform stress profile is from extensive numerical parameter studies aimed at reproducing the tissue motion observed in the pre/post resection MR scans. We found that a nonuniform distribution characterized by the above description was necessary to explain deformation trajectories seen in measurement data.

\section{Results}
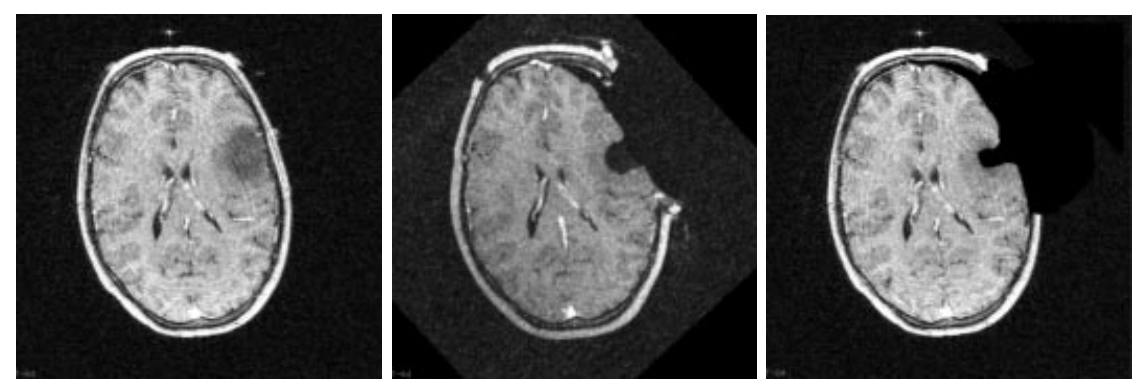

Fig. 3. Co-registered preoperative, intraoperative, and model-based intraoperative update.

Figure 3 is an example of a preoperative image (left), an intraoperative MR update (middle), and a model-based intraoperative update (right). Figure 3 also demonstrates the robustness of the registration algorithm used intraoperatively. Figure 4a.1-a.4 illustrates a point-by-point comparison of anatomic trajectories in Table 1 when subjected to only gravity. Referring to the coordinate system labeled in Figure 1 (right), the largest gravitational component is in the $x$ displacement direction which is also the most satisfying comparison in Figure 4a.1. The most substantial errors occur in surface points along the $y$ displacement direction which corresponds to anterior/posterior movement. In Figure 4b.1b.4 we have included tumor decompression forces which results in a substantial improvement along the $y$ displacement direction. We see better agreement of surface points both anterior and posterior to the decompressed region. 

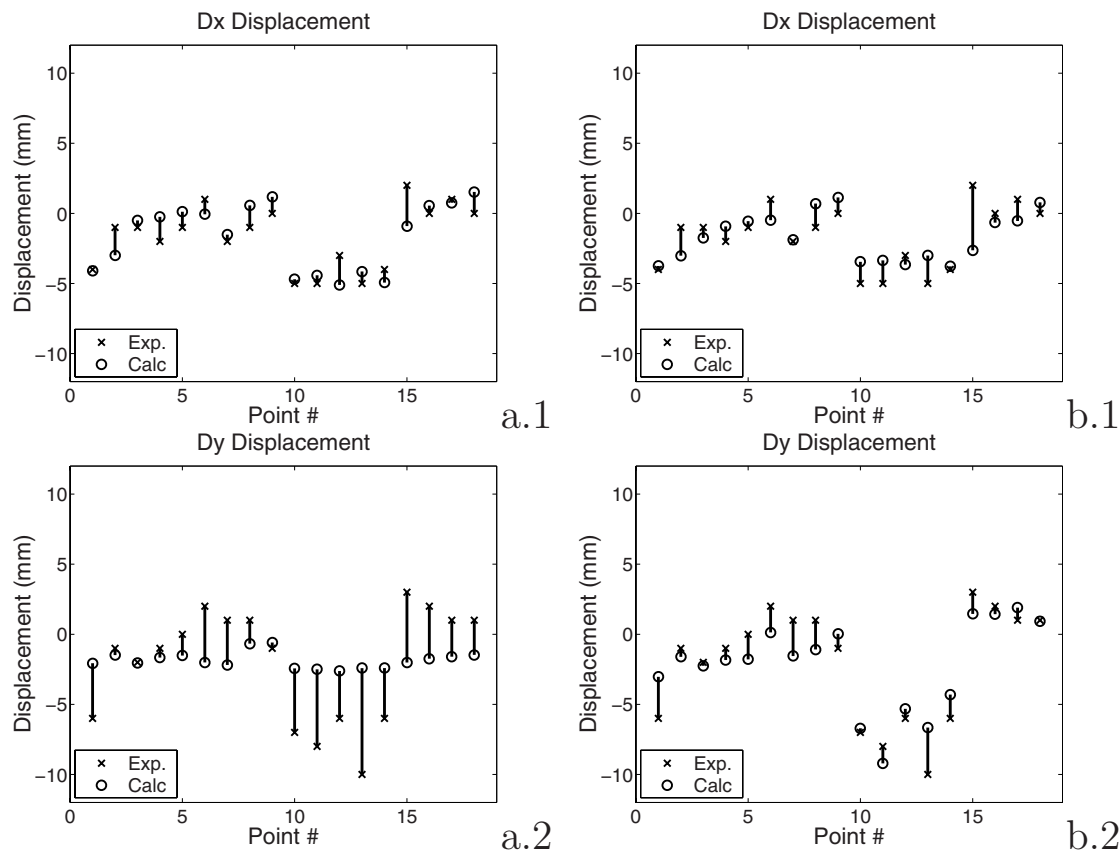

a. 2
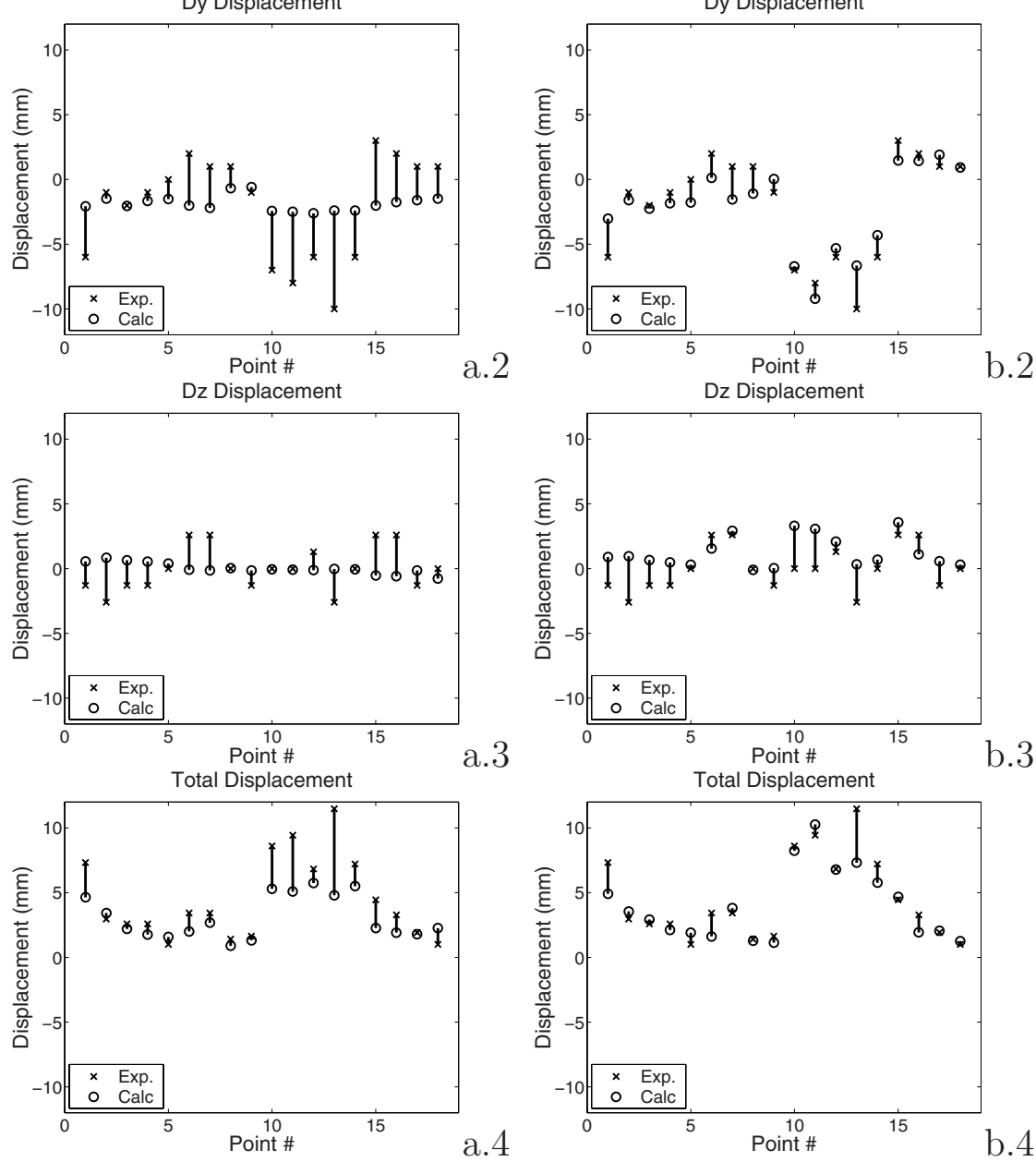

Fig. 4. Tissue trajectory displacements (from Table 1) compared to model calcuations with (a) gravitational forces alone and (b) gravitational forces plus decompression (i.e. indices 1,2,3,4 represent Dx, Dy, Dz, and Total displacements respectively). A good correspondence is achieved when the distance between measured displacement $(\mathrm{X})$ and predicted displacement $(\mathrm{O})$ is small (i.e. line connecting $(\mathrm{X})$ and $(\mathrm{O})$ at each point is small). 


\section{Discussion}

Valuable insight can be gained by observing the measurements reported in Table 1. We see that brain shift is largely limited to the hemisphere of resection in this particular case. Deep seated points such as the third ventricle and the posterior lateral horns move little while the largest subsurface deformation occurs at the highest elevation, point (1). Interestingly we also observe significantly less movement at point (2) which is in close proximity to the falx. A dramatic increase in motion with the largest occurring closest to the resection area is recorded at the surface points (10-18). Here locations both anterior and posterior to the resection move inward toward that region. This is an important observation because given the patient's orientation (Figure $2 \mathrm{~b}$ ) during surgery, the posterior points are moving against the direction of gravity.

In Figure 3, the model-based update is observed to be qualitatively similar to the intraoperative update with the brain surface deforming in the direction of gravity. A different shape and size of the decompressed cavity appears which we attribute to errors in estimating the resection cavity. To estimate the resected tissue volume, the intraoperative cavity was segmented, dilated and positioned centrally over the preoperative MR tumor in AnalyzeAVW - Version 2.5 but ultimately this is an unknown in the current calculation. The trajectories in Figure 4a.1-a.4 show the influence of gravity which is most notably in the $x$ direction. The proportion of gravity-induced motion in the $y$ direction (Figure 4 a.2) is less than in the $x$ direction which makes intuitive sense since the predominant component of gravity is in the $x$ direction. When tumor decompression is added to the calculation in Figure 4b, we see a dramatic improvement in $y$ displacement (Figure 4b.2) with little effect on displacements in the $x$ direction (Figure 4b.1). With respect to the boundary conditions, this agrees with a radial in-plane decompression of the cavity largely restricted to a plane tangent to the brain surface. We note that many numerical simulations were analyzed regarding the distribution of decompressive forces on the cavity boundary with the apportionment presented here capturing the motion best. To our knowledge, this is the first work to correlate tumor-induced stress distributions with in vivo measurement data. Further analysis of Figure 4a-b suggests that gravitational forces are responsible for approximately $40 \%$ of the intraoperative motion while decompressive forces can account for another $30 \%$ (i.e. percentage is based on total displacement comparisons). Assumptions made regarding excision mass, decompression directionality, other intraoperative loads (i.e. retractors) and non-linear effects undoubtedly comprise the remaining motion.

\section{Conclusions}

There is little question that the assumptions made in this analysis need to be investigated further. However, given these limitations, the model shows significant potential in predicting decompression effects from tumor resection. The clinical data, albeit sparse, highlight intraoperative volume deformations and provide interesting insight to the complexity of tissue movement during surgery. The most 


\begin{tabular}{|l|l|}
\hline \hline Computational Operation & Time (min) \\
\hline \hline Problem loading \& B.C. deployment & 2 \\
\hline Build Preconditioner \& 1 Solution & $5-6$ \\
\hline Successive Solutions w/o Rebuilding Preconditioner & $3-4$ \\
\hline Output Solutions & 0.5 \\
\hline \hline
\end{tabular}

Table 2. Computational burden of FEM for a problem with approximately 21,452 nodes and 113,044 elements.

remarkable implication of these results is that with only two temporally-spaced image volumes and with limited knowledge of the actual intraoperative events, a model calculation can predict approximately $70 \%$ of the measured intraoperative motion. However, we must temper this enthusiasm with the realization that we are still uncertain as to the quantity and quality of intraoperative data that can be successfully integrated into a model-updating paradigm. In addition, the computational burdens which accompany finite elements must be considered. Table 2 presents the run times associated with different stages of solution evolution for the problem shown in Figures 1-4 on a Pentium III - $550 \mathrm{MHz}$ PC. On the scale of a several hour neurosurgical procedure, we estimate that effective updates need to be performed every 15-20 minutes which appear feasible given the times reported in Table 2. This implies that the remaining steps (i.e. intraoperative data acquisition, data processing, and image updating) would have to be performed in 7-15 minutes which would also seem to be an attainable goal.

Acknowledgments: This work was supported by National Institutes of Health grant R01-NS33900 awarded by the National Institute of Neurological Disorders and Stroke. AnalyzeAVW - Version 2.5 software was provided in collaboration with the Mayo Foundation, Rochester,MN.

\section{References}

[1] D. L. G. Hill, C. R. Maurer, R. J. Maciunas, J. A. Barwise, J. M. Fitzpatrick, and M. Y. Wang, "Measurement of intraoperative brain surface deformation under a craniotomy," Neurosurgery, vol. 43, no. 3, pp. 514-528, 1998.

[2] D. W. Roberts, A. Hartov, F. E. Kennedy, M. I. Miga, and K. D. Paulsen, "Intraoperative brain shift and deformation: a quantitative clinical analysis of cortical displacements in 28 cases," Neurosurgery, vol. 43, no. 4, pp. 749-760, 1998.

[3] P. M. Black, T. Moriarty, E. Alexander, P. Stieg, E. J. Woodard, P. L Gleason, C. H. Martin, R. Kikinis, R. B. Schwartz, and F. A. Jolesz, "Development and implementation of intraoperative magnetic resonance imaging and its neurosurgical applications," Neurosurgery, vol. 41, no. 4, pp. 831-842, 1997.

[4] V. M. Tronnier, C. R. Wirtz, M. Knauth, G. Lenz, O. Pastyr, M. M. Bonsanto, F. K. Albert, R. Kuth, A. Staubert, W. Schlegel, K. Sartor, and S. Kunze, "Intraoperative diagnostic and interventional magnetic resonance imaging in neurosurgery," Neurosurgery, vol. 40, no. 5, pp. 891-900, 1997.

[5] M. Knauth, C. R. Wirtz, V. M. Tronnier, N. Aras, S. Kunze, and K. Sartor, "Intraoperative MR increases the extent of tumor resection in patients with highgrade gliomas," Am. J. Neuroradiol., vol. 20, no. 9, pp. 1642-1646, 1999. 
[6] M. Knauth, N. Aras, C. R. Wirtz, A. Dorfler, T. Engelhorn, and K. Sartor, "Surgically induced intracranial contrast enhancement: Potential source of diagnostic error in intraoperative MR imaging," Am. J. Neuroradiol., vol. 20, no. 8, pp. 1547-1553, 1999.

[7] P. J. Kelly, "Craniotomy for tumor treatment in an intraoperative magnetic resonance imaging unit - COMMENT," Neurosurgery, vol. 45, no. 3, pp. 432433, 1999.

[8] C. R. Wirtz, M. M. Bonsanto, M. Knauth, V. M. Tronnier, F. K. Albert, A. Staubert, and S. Kunze, "Intraoperative magnetic resonance imaging to update interactive navigation in neurosurgery: Method and preliminary experience," Computer Aided Surgery, vol. 2, pp. 172-179, 1997.

[9] V. M. Tronnier, "Craniotomy for tumor treatment in an intraoperative magnetic resonance imaging unit - COMMENT," Neurosurgery, vol. 45, no. 3, pp. 431-432, 1999.

[10] D. W. Roberts, M. I. Miga, F. E. Kennedy, A. Hartov, and K. D. Paulsen, "Intraoperatively updated neuroimaging using brain modeling and sparse data," Neurosurgery, vol. 45, no. 5, pp. 1199-1207, 1999.

[11] M. I. Miga, K. D. Paulsen, P. J. Hoopes, F. E. Kennedy, A. Hartov, and D. W. Roberts, "In vivo quantification of a homogeneous brain deformation model for updating preoperative images during surgery," IEEE Transactions on Biomedical Engineering, vol. 47, no. 2, pp. 266-273, 2000.

[12] M. I. Miga, K. D. Paulsen, J. M. Lemery, S. D. Eisner, A. Hartov, F. E. Kennedy, and D. W. Roberts, "Model-updated image guidance: Initial clinical experiences with gravity-induced brain deformation," IEEE Trans. Med. Imaging, vol. 18, no. 10, pp. 866-874, 1999.

[13] Wirtz C. R., V. M. Tronnier, F. K. Albert, M. Knauth, M. M. Bonsanto, A. Staubert, O. Pastyr, and S. Kunze, "Modified headholder and operating table for intra-operative MRI in neurosurgery," Neurol. Res., vol. 20, no. 7, pp. 658-661, 1998.

[14] Wirtz C. R., V. M. Tronnier, M. M. Bonsanto, A. Staubert, F. K. Albert, , and S. Kunze, "Image-guided neurosurgery with intraoperative MRI: update of frameless stereotaxy and radicality control," Stereotact. Funct. Neurosurg., vol. 68, pp. 39-43, 1997.

[15] M. A. Biot, "General theory of three-dimensional consolidation," J. App. Physics, vol. 12, pp. 155-164, 1941.

[16] K. D. Paulsen, M. I. Miga, F. E. Kennedy, P. J. Hoopes, A. Hartov, and D. W. Roberts, "A computational model for tracking subsurface tissue deformation during stereotactic neurosurgery," IEEE Trans. Biomed. Eng., vol. 46, no. 2, pp. 213-225, 1999.

[17] M. I. Miga, K. D. Paulsen, and F. E. Kennedy, "Von neumann stability analysis of Biot's general two-dimensional theory of consolidation," Int. J. of Num. Methods in Eng., vol. 43, pp. 955-974, 1998.

[18] M. I. Miga, K. D. Paulsen, P. J. Hoopes, F. E. Kennedy, A. Hartov, and D. W. Roberts, "In-vivo analysis of heterogeneous brain deformation computations for model-updated image guidance," Computer Methods in Biomechanics and Biomedical Engineering, vol. 3, pp. 129-146, 2000.

[19] M. I. Miga, K. D. Paulsen, P. J. Hoopes, F. E. Kennedy, A. Hartov, and D. W. Roberts, "In vivo modeling of interstitial pressure in the brain under surgical load using finite elements," ASME J. Biomech. Eng., (in press), 2000. 